\title{
RNA Sequencing Provides Evidence for Pathogenicity of a Novel CHEK2 Splice Variant (C.1009-7T>G)
}

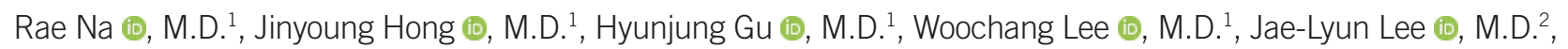
Sail Chun (1), M.D. ${ }^{1}$, and Won-Ki Min (0), M.D. ${ }^{1}$

Departments of ${ }^{1}$ Laboratory Medicine and ${ }^{2}$ Oncology, Asan Medical Center, University of Ulsan College of Medicine, Seoul, Korea

\section{Dear Editor,}

Prostate cancer is common worldwide and is the fourth most common cancer in Korean men [1]. Although the exact cause of prostate cancer is unknown, its incidence is higher among certain ethnicities, in the elderly, and in individuals with a family history [2]. Inherited variants in several DNA repair genes, including BRCA1, BRCA2, CHEK2, and ATM, were recently associated with responses to poly (ADP-ribose) polymerase (PARP) inhibition in prostate, ovarian, and breast cancers [3]. Loss of function of these genes is associated with more aggressive prostate cancer, and a clinical trial on the PARP inhibitor olaparib for metastatic prostate cancer is ongoing [3]. CHEK2 is a tumor suppressor that plays an important role in genome integrity and the cellular response to DNA damage through the phosphorylation of several substrates, including BRCA1 and TP53 [4, 5]. CHEK2 is currently included in many next-generation sequencing (NGS) panels targeting various hereditary cancers, and new studies are continually being published. We identified a novel splice variant of CHEK2 by NGS and confirmed its impact on splicing by RNA sequencing.

In 2021, a 70-year-old man undergoing chemotherapy for metastatic prostate cancer without a known family history showed a continuous increase in prostate-specific antigen (PSA). After obtaining informed consent, NGS was performed for 171 here- ditary tumor-related genes for further evaluation and management at Asan Medical Center, Seoul, Korea. Genetic testing was performed as a clinical laboratory test and not with a research objective; approval from the Institutional Review Board was exempted. Genomic DNA was extracted from peripheral blood leukocytes using a customized target enrichment kit (Dxome, Seongnam, Korea), and NGS was conducted on a MiSeqDx instrument (Illumina, San Diego, CA, USA) using a MiSeqDx V2 sequencing kit (Illumina).

The mean coverage depth was 554.2x. Except for one intronic variant in CHEK2, 256 variants were predicted to be benign or likely benign. The suspected variant, NM_007194.4 (CHEK2): c. 1009-7T > G, was heterozygous, with a variant allele frequency of $50.2 \%$ (Fig. 1). This variant has conflicting interpretations of pathogenicity in ClinVar but is not reported in the Human Gene Mutation Database. According to the 2015 American College of Medical Genetics (ACMG) guidelines, given its rarity in the general population, only a designation of PM2 (absent from controls) could be applied to the variant [6]. In in-silico splice-site prediction, the Database of Splicing Consensus Single-Nucleotide Variants adaptive boosting score was 0.387 , and the random forest score was 0.288. In NetGene2-based splicesite prediction, the possibility of a splicing change was predicted to be low; thus, the designation of PP3 (multiple lines of compu-
Received: July 23, 2021

Revision received: September 22, 2021

Accepted: November 26, 2021

Corresponding author: Woochang Lee, M.D.

Department of Laboratory Medicine, Asan Medical Center, University of Ulsan College of Medicine, 88 Olympic-ro 43-gil, Songpa-gu, Seoul 05505, Korea

Tel.: +82-2-3010-4506, Fax: +82-2-478-0884

E-mail: wlee1@amc.seoul.kr 


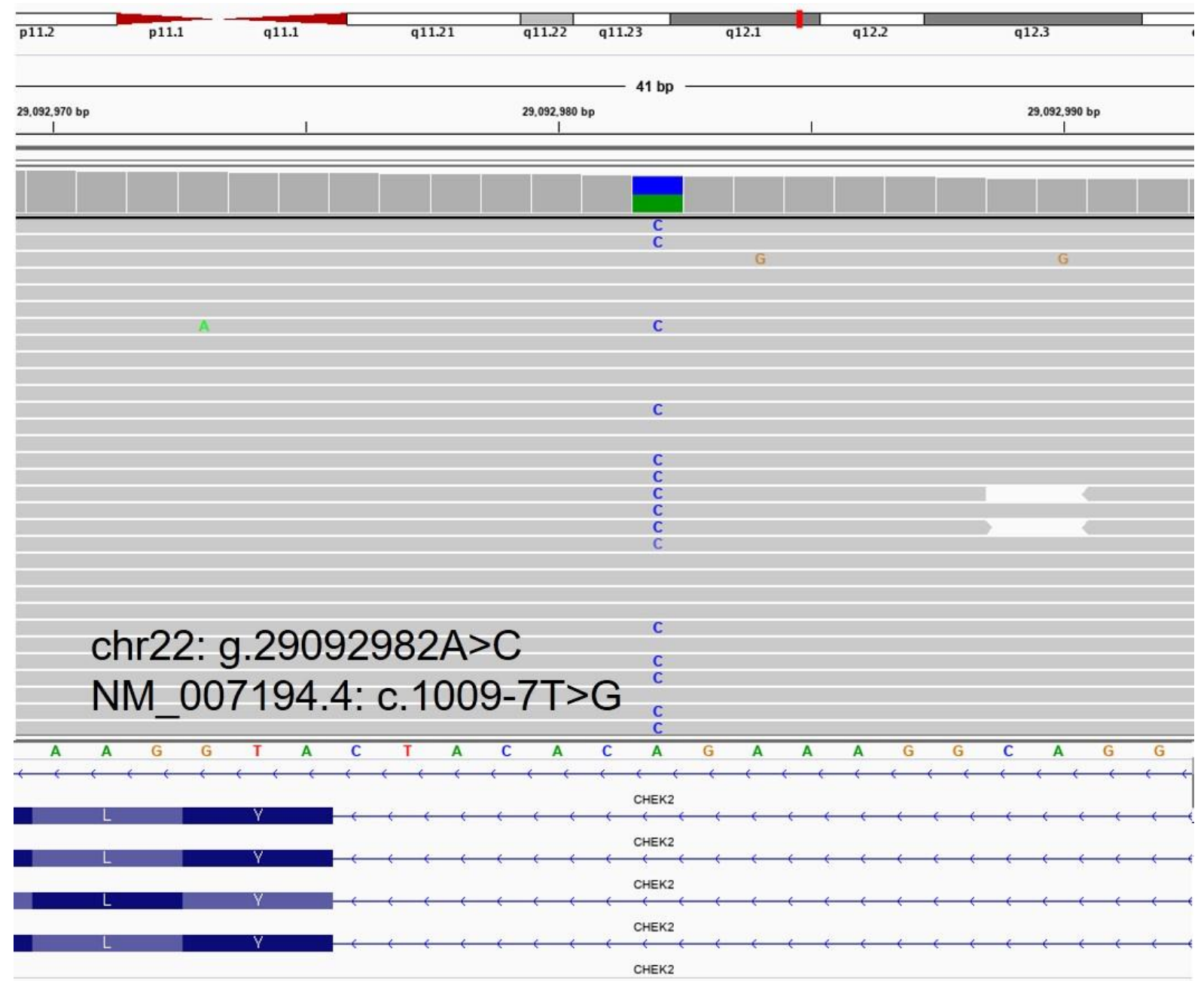

Fig. 1. Next-generation sequencing revealed a variant of CHEK2, involving a substitution of thymine (T) for guanine (G) at the intron located -7 from the acceptor site (c.1009T > G).

tational evidence supporting a deleterious effect of the gene) was not applicable [7]. The variant was classified as a variant of uncertain significance (VUS) [6].

Given the implication of CHEK2 in prostate cancer and the CHEK2 variant being located close to the splicing acceptor site, RNA sequencing was performed to confirm the impact of the variant at the RNA level. RNA was extracted from leukocytes of a healthy volunteer and the patient using High Pure RNA Isolation Kit (Roche, Indianapolis, IN, USA) and reverse-transcribed using the RevertAid First Strand cDNA Synthesis kit (Thermo Fisher Scientific, Waltham, MA, USA). Exon 10 skipping with an 87-bp deletion was detected in the patient. This variant, represented as NM_007194.4(CHEK2):c.1009_1095del (Fig. 2), has not been reported previously. The in-frame deletion is in a nonrepeat region that changes the protein length; thus, the desig- nation of PM4 could be applied [6]. ACMG standards do not provide detailed guidance on how functional evidence should be evaluated for a PS3 classification (functional studies supportive of a damaging effect on the gene or gene product); thus, application to the in-frame deletion may be controversial [8]. No deletions in exon 10 are reported in current disease databases. Exon 10 encodes an ATP-binding site located in the kinase domain of CHEK2, as reported in UniProt. As this site plays a critical role in the kinase function of $\mathrm{CHEK} 2$, the deletion in exon 10 may affect protein function [9]. PM1 (critical and well-established functional domain) may be additionally applied, which leads to reclassification of the variant as "likely pathogenic" [6]. We recommended additional NGS testing to the patient's family members, which have not yet been conducted.

As clinical sequencing is mostly performed at the DNA level, 

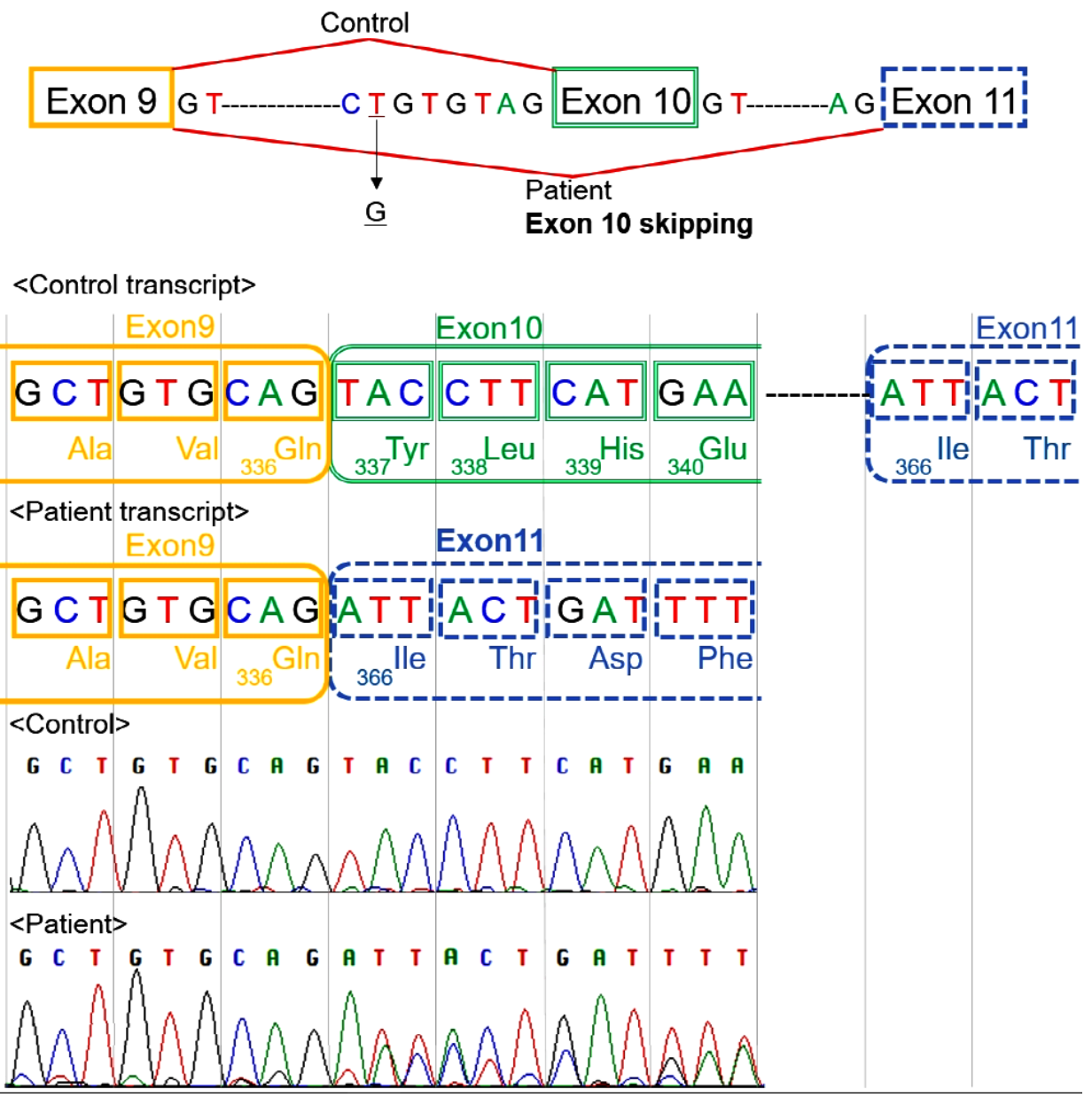

Fig. 2. RNA sequencing revealed exon 10 skipping. This variant is represented as NM_007194.4(CHEK2):c.1009_1095del.

disease-causing splicing variants may be underestimated [10]. The presence of numerous VUS compromises a precise assessment of the risk associated with particular tumor types [9]. RNA sequencing was performed to find evidence of PS3 in a suspected splice variant, which confirmed an in-frame deletion caused by exon 10 skipping. The VUS was reclassified as PM1 and PM4, rather than PS3. RNA sequencing should be more actively considered to provide additional evidence for the determination of pathogenicity categories other than PS3.

\section{ACKNOWLEDGEMENTS}

None.

\section{AUTHOR CONTRIBUTIONS}

$\mathrm{Na} R$ wrote the manuscript. Lee J managed the patient and provided the clinical information. Hong J, Gu H, Lee W, and Min W contributed to the interpretation of the results, and the writing and revision of the manuscript.

\section{CONFLICTS OF INTEREST}

No potential conflicts of interest relevant to this paper are reported.

\section{RESEARCH FUNDING}

None declared.

\section{ORCID}

Rae Na Jinyoung Hong Hyunjung Gu Woochang Lee https://orcid.org/0000-0002-9474-6449 https://orcid.org/0000-0001-8855-7900 https://orcid.org/0000-0001-8673-7326 https://orcid.org/0000-0003-3956-6397 
$\mathrm{Na} \mathrm{R}$, et al.

Novel CHEK2 splice variant (C.1009-7T>G)

Jae-Lyun Lee

https://orcid.org/0000-0002-9420-7162

Sail Chun

https://orcid.org/0000-0002-5792-973X

Won-Ki Min

https://orcid.org/0000-0002-5158-2130

\section{REFERENCES}

1. National Cancer Information Center. National Cancer Registration Program, Annual report of cancer statistics in Korea in 2018. http://www. cancer.go.kr (Updated on 19th November 2021).

2. Wu Y, Yu H, Zheng SL, Na R, Mamawala M, Landis T, et al. A comprehensive evaluation of CHEK2 germline mutations in men with prostate cancer. Prostate 2018;78:607-15.

3. de Bono J, Mateo J, Fizazi K, Saad F, Shore N, Sandhu S, et al. Olaparib for metastatic castration-resistant prostate cancer. N Engl J Med 2020; 382:2091-102.

4. Delimitsou A, Fostira F, Kalfakakou D, Apostolou P, Konstantopoulou I, Kroupis C, et al. Functional characterization of CHEK2 variants in a SaCcharomyces cerevisiae system. Hum Mutat 2019;40:631-48.
5. Cybulski C, Górski B, Huzarski T, Masojć B, Mierzejewski M, Debniak T, et al. CHEK2 is a multiorgan cancer susceptibility gene. Am J Hum Genet 2004;75:1131-5.

6. Richards S, Aziz N, Bale S, Bick D, Das S, Gastier-Foster J, et al. Standards and guidelines for the interpretation of sequence variants: a joint consensus recommendation of the American College of Medical Genetics and Genomics and the Association for Molecular Pathology. Genet Med 2015;17:405-24.

7. Jian $\mathrm{X}$, Boerwinkle $\mathrm{E}$, Liu X. In silico prediction of splice-altering single nucleotide variants in the human genome. Nucleic Acids Res 2014;42: 13534-44.

8. Brnich SE, Abou Tayoun AN, Couch FJ, Cutting GR, Greenblatt MS, Hei nen $C D$, et al. Recommendations for application of the functional evidence PS3/BS3 criterion using the ACMG/AMP sequence variant interpretation framework. Genome Med 2019;12:3.

9. Stolarova L, Kleiblova P, Janatova M, Soukupova J, Zemankova P, Macurek L, et al. CHEK2 germline variants in cancer predisposition: stalemate rather than checkmate. Cells 2020;9:2675.

10. Anna A and Monika G. Splicing mutations in human genetic disorders: examples, detection, and confirmation. J Appl Genet 2018;59:253-68. 